\title{
Reversion from prediabetes to normoglycaemia and risk of cardiovascular disease and mortality: the Whitehall II cohort study
}

\author{
Dorte Vistisen ${ }^{1}$ (1) - Mika Kivimäki ${ }^{2}$ - Leigh Perreault ${ }^{3} \cdot$ Adam Hulman $^{4,5,6}$ - Daniel R. Witte ${ }^{4,5,6}$ - Eric J. Brunner ${ }^{2}$. \\ Adam Tabák ${ }^{2,7} \cdot$ Marit E. Jørgensen ${ }^{1,8} \cdot$ Kristine Færch $^{1}$
}

Received: 28 February 2019 / Accepted: 10 April 2019 / Published online: 23 May 2019

(C) The Author(s) 2019

\begin{abstract}
Aims/hypothesis Reversion from prediabetes to normoglycaemia is accompanied by an improvement in cardiovascular risk factors, but it is unclear whether this translates into a reduction in risk of cardiovascular disease (CVD) events or death. Hence, we studied the probability of reversion from prediabetes to normoglycaemia and the associated risk of future CVD and death using data from the Whitehall II observational cohort study.

Methods Three glycaemic criteria for prediabetes (fasting plasma glucose [FPG] 5.6-6.9 mmol/l, $2 \mathrm{~h}$ plasma glucose [2hPG] 7.8-11.0 mmol/1, and $\mathrm{HbA}_{1 \mathrm{c}} 39-47 \mathrm{mmol} / \mathrm{mol}$ [5.7-6.4\%]) were assessed in 2002-2004 and 2007-2009 for 5193 participants free of known diabetes at enrolment. Among participants with prediabetes in the first examination, we calculated the probability of reversion to normoglycaemia by re-examination according to each glycaemic criterion. Poisson regression analysis was used to estimate and compare incidence rates of a composite endpoint of a CVD event or death in participants with prediabetes who did vs did not revert to normoglycaemia. Analyses were adjusted for age, sex, ethnicity and previous CVD.

Results Based on the FPG criterion, 820 participants had prediabetes and $365(45 \%)$ of them had reverted to normoglycaemia in 5 years. The corresponding numbers were 324 and 120 (37\%) for the $2 \mathrm{hPG}$ criterion and 1709 and $297(17 \%)$ for the $\mathrm{HbA}_{1 \mathrm{c}}$ criterion. During a median follow-up of 6.7 (interquartile range 6.3-7.2) years, 668 events of non-fatal CVD or death occurred among the 5193 participants. Reverting from $2 \mathrm{hPG}$-defined prediabetes to normoglycaemia vs remaining prediabetic or progressing to diabetes was associated with a halving in event rate ( $12.7 \mathrm{vs} 29.1$ per 1000 person-years, $p=0.020)$. No association with event rate was observed for reverting from FPG-defined (18.6 vs 18.2 per 1000 person-years, $p=0.910)$ or HbA $_{1 c^{-}}$ defined prediabetes to normoglycaemia (24.5 vs 22.9 per 1000 person-years, $p=0.962$ ).

Conclusions/interpretation Most people with $\mathrm{HbA}_{1 \mathrm{c}}$-defined prediabetes remained prediabetic or progressed to diabetes during 5 years of follow-up. In contrast, reversion to normoglycaemia was frequent among people with FPG- or $2 \mathrm{hPG}$-defined prediabetes. Only reversion from $2 \mathrm{hPG}$-defined prediabetes to normoglycaemia was associated with a reduction in future risk of CVD and death.
\end{abstract}

Electronic supplementary material The online version of this article (https://doi.org/10.1007/s00125-019-4895-0) contains peer-reviewed but unedited supplementary material, which is available to authorised users.

\section{Dorte Vistisen \\ dorte.vistisen@ regionh.dk \\ Mika Kivimäki \\ m.kivimaki@ucl.ac.uk}

1 Department of Clinical Epidemiology, Steno Diabetes Center Copenhagen, Niels Steensens Vej 6, DK-2820 Gentofte, Denmark

2 Department of Epidemiology and Public Health, University College London, 1-19 Torrington Place, London WC1E 7HB, UK
Department of Medicine, Division of Endocrinology, Metabolism and Diabetes, University of Colorado, Anschutz Medical Campus, Aurora, CO, USA

4 Department of Public Health, Aarhus University, Aarhus, Denmark

5 Danish Diabetes Academy, Odense, Denmark

6 Steno Diabetes Center Aarhus, Aarhus, Denmark

7 First Department of Medicine, Faculty of Medicine, Semmelweis University, Budapest, Hungary

8 The Research Department for Health and Morbidity in the Population, Southern Denmark University, Copenhagen, Denmark 


\section{Research in context}

\section{What is already known about this subject?}

- Prediabetes is associated with an increased risk of cardiovascular disease (CVD) and death

- Reversion from prediabetes to normoglycaemia has been shown to improve cardiovascular risk factors

\section{What is the key question?}

- Is reversion from prediabetes to normoglycaemia associated with a lower risk of CVD and death?

\section{What are the new findings?}

- Individuals reverting from fasting glucose- or from $\mathrm{HbA}_{1 c}$-defined prediabetes to normoglycaemia were not at reduced risk of future CVD or death

- Reversion to normoglycaemia from prediabetes defined by $2 \mathrm{~h}$ glucose was associated with a halving in future risk of CVD and death

\section{How might this impact on clinical practice in the foreseeable future?}

- People with elevated $2 \mathrm{~h}$ glucose seem to have a strong potential to decrease their CVD risk through reducing glucose levels, and therefore this group should be identified for early preventive strategies

Keywords $2 \mathrm{~h}$ Plasma glucose $\cdot$ Cardiovascular disease $\cdot$ Fasting plasma glucose $\cdot \mathrm{HbA}_{1 \mathrm{c}} \cdot$ Mortality $\cdot$ Normoglycaemia . Prediabetes $\cdot$ Reversion

\author{
Abbreviations \\ 2hPG $2 \mathrm{~h}$ plasma glucose \\ CVD Cardiovascular disease \\ FPG Fasting plasma glucose \\ IEC International Expert Committee
}

\section{Introduction}

Prediabetes increases the risk of cardiovascular disease (CVD) and mortality $[1,2]$, and reversion from prediabetes to normoglycaemia is related to an improvement in a range of cardiovascular risk factors [3]. However, whether reversion translates into a reduction in risk of CVD or death has yet to be determined. To fill this knowledge gap, the current analysis explored the probability of reversion from prediabetes to normoglycaemia from different definitions of prediabetes and the associated risk of future CVD and death in a longitudinal follow-up of the well-described Whitehall II observational cohort study.

\section{Methods}

Study population The Whitehall II study is an occupational cohort of 10,308 British civil servants who have been followed up with clinical examinations every 5 years since 1985 [4]. The current analysis utilised data from phase 7 (2002-2004) and phase 9 (2007-2009), when fasting plasma glucose (FPG),
$2 \mathrm{~h}$ plasma glucose $(2 \mathrm{hPG})$ and $\mathrm{HbA}_{1 \mathrm{c}}$ were measured. From the 6967 participants at phase 7, we excluded 671 (9.6\%) who did not participate at phase 9, $392(5.6 \%)$ with known diabetes at phase $7,684(9.8 \%)$ who could not be classified with normoglycaemia, prediabetes or diabetes at both phases on at least one criterion, and $27(0.4 \%)$ with screen-detected diabetes according to all the three criteria at phase 7, leaving 5193 (74.5\%) of the phase 7 participants for analysis (ESM Fig. 1). For the analyses of FPG and 2hPG, participants had to have fasted for $\geq 8 \mathrm{~h}$ before the clinical examinations. The University College London Ethics Committee reviewed and approved the study. Written informed consent was obtained from all participants at each study phase.

Measurements and definitions Participants underwent a standard $75 \mathrm{~g}$ OGTT with measurement of FPG and 2hPG using the glucose oxidase method [4]. $\mathrm{HbA}_{1 \mathrm{c}}$ was measured in whole blood, drawn into BD Vacutainers (Becton Dickinson, Winnersh, UK), using the validated Tosoh G8 highperformance ion-exchange liquid chromatography platform (Tosoh Bioscience, Tessenderlo, Belgium).

Prediabetes was defined as FPG 5.6-6.9 mmol/1, 2hPG 7.8$11.0 \mathrm{mmol} / \mathrm{l}$ and/or $\mathrm{HbA}_{1 \mathrm{c}} 39-47 \mathrm{mmol} / \mathrm{mol}(5.7-6.4 \%$ ) according to criteria from the ADA [5]. For each of the three criteria, normoglycaemia and diabetes were defined as values below and above the cut-off points for prediabetes, respectively. Diabetes could also be diagnosed by a doctor outside the study.

The primary outcome was a composite endpoint of a CVD event or death between 2007-2009 (phase 9) and the end of 
follow-up (30 June 2015). Adjudicated CVD events included fatal and non-fatal myocardial infarction and stroke [2]. Allcause mortality was obtained from the NHS Central Registry, which provided information on the cause and date of death.

Statistical analysis All analyses were conducted separately for the three glycaemic criteria, in accordance with the guidelines from ADA [5] and the International Expert Committee (IEC) [6], which suggest that the different criteria to define prediabetes and diabetes should not be combined. For each criterion, participants with screen-detected diabetes at baseline by the given glycaemic criterion were excluded.

Among individuals with prediabetes at phase 7, we calculated the probability of reversion to normoglycaemia at the phase 9 re-examination approximately 5 years later. Poisson regression analysis with $\log$ (person time) as offset was used to estimate and compare incidence rates of future CVD or death in individuals with prediabetes who did or did not revert to normoglycaemia. The follow-up period of each participant was split into 1 year age bands to account for the nonconstant effect of age over time on CVD risk and mortality. Analyses were adjusted for age, sex, ethnicity and previous CVD. A complete case approach was used.

All participants with $2 \mathrm{hPG}$ available also had measurements of FPG and $\mathrm{HbA}_{1 \mathrm{c}}$ (but not vice versa). Among participants with $2 \mathrm{hPG}$-defined prediabetes and elevated FPG and/ or $\mathrm{HbA}_{1 \mathrm{c}}$, we compared event rates between participants who did or did not revert to normoglycaemia on FPG and/or $\mathrm{HbA}_{1 \mathrm{c}}$, without normalising $2 \mathrm{hPG}$ (ESM Fig.1).

We further estimated the associations of changes in FPG, $2 \mathrm{hPG}$ or $\mathrm{HbA}_{1 \mathrm{c}}$ from phase 7 to phase 9 with risk of future CVD or death, excluding participants with known diabetes at phase 9 because they were likely to be receiving treatment.

Statistical analyses were performed in R, version 3.4.1 (R Foundation for Statistical Computing, Vienna, Austria).

\section{Results}

The study population was predominantly men (73\%) and of white ethnicity (93\%), 769 (15\%) had pre-existing CVD, and the average age was 60 years (range 50-73 years) at the first clinical examination (phase 7). During a median follow-up of 6.7 (interquartile range 6.3-7.2) years, 668 events of non-fatal CVD or death occurred among the 5193 participants. Comparing normoglycaemia vs prediabetes by each glycaemic criterion (Table 1), more men than women had prediabetes defined by FPG whereas no sex difference was observed for prediabetes defined by $2 \mathrm{hPG}$ or $\mathrm{HbA}_{1 \mathrm{c}}$. Participants with $2 \mathrm{hPG}$ - or $\mathrm{HbA}_{1 c^{-}}$, but not FPG-defined prediabetes were older $(p<0.001)$ and more likely to be of nonwhite ethnicity $(p \leq 0.044)$ compared with their normoglycaemic counterparts.
FPG criterion Among 820 participants with FPG-defined prediabetes at baseline, $365(45 \%)$ reverted to normoglycaemia and 111 (14\%) progressed to diabetes at the 5 year re-examination. From the 5 year re-examination, median (interquartile range) follow-up time was 6.7 (6.3-7.2) years, during which $96(12 \%)$ developed CVD or died. Reverting from prediabetes to normoglycaemia was not associated with a difference in rate of developing CVD or dying vs remaining prediabetic or progressing to diabetes (18.6 vs 18.2 per 1000 personyears, $p=0.962)$ (Fig. 1a, ESM Table 1).

2hPG criterion Among 324 participants with 2hPG-defined prediabetes at baseline, $120(37 \%)$ reverted to normoglycaemia and $73(23 \%)$ progressed to diabetes after 5 years. Follow-up time for CVD and death was 6.7 (6.4-7.2) years, during which $47(15 \%)$ developed CVD or died. Reverting from prediabetes to normoglycaemia was associated with a halving of the event rate vs remaining prediabetic/progressing to diabetes (12.7 vs 29.1 per 1000 person-years, $p=0.020$ ), and was largely unchanged after adjustment for age, sex, ethnicity and previous CVD (Fig. 1a, ESM Table 1).

Among the 324 participants with prediabetes at baseline, 220 also had prediabetes according to the FPG and/or $\mathrm{HbA}_{1 \mathrm{c}}$ criteria. At the 5 year re-examination, $21(10 \%)$ of these had reverted to normoglycaemia according to the FPG and/or $\mathrm{HbA}_{1 \mathrm{c}}$ criteria but not according to the $2 \mathrm{hPG}$ criterion. However, improvement in FPG or $\mathrm{HbA}_{1 \mathrm{c}}$ without improvement in $2 \mathrm{hPG}$ was not associated with a lower event rate (32.1 vs 28.8 per 1000 person-years, respectively, $p=0.835$ ).

$\mathrm{HbA}_{1 c}$ criterion Among 1709 participants with $\mathrm{HbA}_{1 \mathrm{c}}$-defined prediabetes at baseline, 297 (17\%) reverted to normoglycaemia and 233 (14\%) progressed to diabetes after 5 years. Follow-up time for an event was 6.7 (6.3-7.2) years, during which 258 (15\%) developed CVD or died. Reverting from prediabetes to normoglycaemia was not associated with a difference in rate of developing CVD or dying vs remaining prediabetic/progressing to diabetes ( 24.5 vs 22.9 per 1000 person-years, $p=0.962$ ) (Fig. 1a, ESM Table 1).

The analysis of absolute changes in FPG, $2 \mathrm{hPG}$ or $\mathrm{HbA}_{1 \mathrm{c}}$ from phase 7 to phase 9 confirmed the results (Fig. 1b, ESM Table 2).

\section{Discussion}

In this cohort study, reversion from 2hPG-defined prediabetes to normoglycaemia was associated with an approximately $50 \%$ lower risk of a CVD event or death compared with remaining with prediabetes or progressing to diabetes. Despite prediabetes being a known risk factor for $\mathrm{CVD}$, we found that reversion from FPG- or $\mathrm{HbA}_{1 \mathrm{c}}$-defined prediabetes to normoglycaemia was not associated with a lower risk of 
Table 1 Characteristics of study participants at the first clinical examination (phase 7) by glycaemic criterion

\begin{tabular}{|c|c|c|c|c|c|c|}
\hline \multirow[t]{2}{*}{ Characteristic } & \multicolumn{2}{|l|}{ FPG criterion } & \multicolumn{2}{|l|}{$2 \mathrm{hPG}$ criterion } & \multicolumn{2}{|l|}{$\mathrm{HbA}_{1 \mathrm{c}}$ criterion } \\
\hline & Normoglycaemia & Prediabetes & Normoglycaemia & Prediabetes & Normoglycaemia & Prediabetes \\
\hline$n$ & 2130 & 820 & 2154 & 324 & 3337 & 1709 \\
\hline Men $(\%)$ & $70.0(68.1,72.0)$ & $83.9(81.2,86.4)$ & $75.7(73.8,77.5)$ & $76.2(71.2,80.8)$ & $72.6(71.0,74.1)$ & $73.6(71.5,75.7)$ \\
\hline White ethnicity (\%) & $92.5(91.3,93.6)$ & $93.2(91.2,94.8)$ & $93.1(91.9,94.1)$ & $89.8(86.0,92.9)$ & $96.3(95.6,96.9)$ & $89.4(87.9,90.8)$ \\
\hline Age (years) & $60.2 \pm 5.8$ & $60.6 \pm 5.8$ & $59.6 \pm 5.6$ & $62.0 \pm 6.1$ & $60.2 \pm 5.7$ & $61.6 \pm 5.9$ \\
\hline BMI $\left(\mathrm{kg} / \mathrm{m}^{2}\right)$ & $24.0 \pm 3.1$ & $24.9 \pm 3.2$ & $24.0 \pm 3.1$ & $24.9 \pm 3.1$ & $23.9 \pm 3.0$ & $24.6 \pm 3.3$ \\
\hline Total cholesterol (mmol/l) & $5.7 \pm 1.0$ & $5.8 \pm 1.0$ & $5.7 \pm 1.0$ & $5.7 \pm 1.0$ & $5.7 \pm 1.0$ & $5.8 \pm 1.0$ \\
\hline HDL-cholesterol (mmol/l) & $1.6 \pm 0.5$ & $1.5 \pm 0.4$ & $1.6 \pm 0.4$ & $1.5 \pm 0.4$ & $1.6 \pm 0.5$ & $1.5 \pm 0.4$ \\
\hline LDL-cholesterol (mmol/l) & $3.5 \pm 0.9$ & $3.6 \pm 0.9$ & $3.6 \pm 0.9$ & $3.6 \pm 0.9$ & $3.5 \pm 0.9$ & $3.6 \pm 0.9$ \\
\hline Triacylglycerols (mmol/l) & $1.1(0.8-1.5)$ & $1.2(0.9-1.7)$ & $1.1(0.8-1.5)$ & $1.3(0.9-1.85)$ & $1.1(0.8-1.5)$ & $1.2(0.9-1.8)$ \\
\hline Systolic BP (mmHg) & $126.0 \pm 16.3$ & $131.2 \pm 16.4$ & $125.6 \pm 15.7$ & $130.6 \pm 16.7$ & $126.3 \pm 16.1$ & $129.0 \pm 16.3$ \\
\hline Diastolic BP (mmHg) & $73.6 \pm 10.3$ & $76.5 \pm 10.1$ & $73.7 \pm 10.3$ & $75.0 \pm 10.2$ & $73.8 \pm 10.4$ & $74.9 \pm 10.2$ \\
\hline FPG $(\mathrm{mmol} / \mathrm{l})$ & $5.0 \pm 0.3$ & $5.9 \pm 0.3$ & $5.3 \pm 0.5$ & $5.6 \pm 0.7$ & $5.2 \pm 0.5$ & $5.5 \pm 0.6$ \\
\hline $2 \mathrm{hPG}(\mathrm{mmol} / \mathrm{l})$ & $5.9 \pm 1.5$ & $6.9 \pm 2.0$ & $5.7 \pm 1.1$ & $8.9 \pm 0.9$ & $5.9 \pm 1.5$ & $6.6 \pm 1.9$ \\
\hline $\mathrm{HbA}_{1 \mathrm{c}}(\mathrm{mmol} / \mathrm{mol})$ & $33.0 \pm 4.0$ & $35.0 \pm 5$ & $33.0 \pm 4$ & $36.0 \pm 5$ & $35.0 \pm 3.0$ & $42.0 \pm 2.0$ \\
\hline $\mathrm{HbA}_{1 \mathrm{c}}(\%)$ & $5.2 \pm 0.4$ & $5.4 \pm 0.4$ & $5.2 \pm 0.4$ & $5.4 \pm 0.4$ & $5.4 \pm 0.3$ & $6.0 \pm 0.2$ \\
\hline Previous CVD $(\%)$ & $12.4(11.1,13.9)$ & $17.1(14.6,19.8)$ & $12.5(11.2,14)$ & $18.5(14.4,23.2)$ & $11.4(10.4,12.6)$ & $18.0(16.2,19.9)$ \\
\hline Family history of DM (\%) & $9.3(8.1,10.6)$ & $12.3(10.1,14.8)$ & $9.1(7.9,10.4)$ & $10.0(6.9,13.8)$ & $8.4(7.4,9.4)$ & $12.2(10.7,13.9)$ \\
\hline Current smoker (\%) & $8.0(6.9,9.2)$ & $7.9(6.2,10.0)$ & $7.7(6.6,8.9)$ & $4.6(2.6,7.5)$ & $6.0(5.2,6.9)$ & $9.2(7.9,10.7)$ \\
\hline Alcohol intake (units/week) & $8.0(2.0-16.0)$ & $12.0(5.0-21.0)$ & $10.0(4.0-18.0)$ & $9.0(3.0-17.0)$ & $10.0(4.0-18.0)$ & $8.0(2.0-16.0)$ \\
\hline Antihypertensive treatment (\%) & $19.5(17.9,21.3)$ & $28.2(25.1,31.4)$ & $18.4(16.8,20.1)$ & $33.3(28.2,38.8)$ & $17.9(16.6,19.3)$ & $26.6(24.5,28.8)$ \\
\hline Lipid-lowering treatment (\%) & $7.8(6.7,9.0)$ & $13.4(11.2,15.9)$ & $7.5(6.4,8.7)$ & $15.4(11.7,19.8)$ & $6.9(6.0,7.8)$ & $13.4(11.8,15.1)$ \\
\hline
\end{tabular}

Data are means \pm SD, medians ( $25-75 \%$ percentiles; IQR) or proportions $(95 \% \mathrm{CI})$

$\mathrm{HbA}_{1 \mathrm{c}}$ : normoglycaemia: $<39 \mathrm{mmol} / \mathrm{mol}(5.7 \%)$, prediabetes 39-47 mmol/mol (5.7-6.4\%), Fasting plasma glucose: normoglycaemia: $<5.6 \mathrm{mmol} / \mathrm{l}$, prediabetes 5.6-6.9 mmol/1, 2hPG: normoglycaemia: $<7.8 \mathrm{mmol} / 1$, prediabetes $7.8-11.0 \mathrm{mmol} / \mathrm{l}$

DM, diabetes mellitus

CVD or death. Outside pregnancy, the OGTT has largely been eliminated for diagnosing diabetes, particularly with the recent widespread standardisation of the $\mathrm{HbA}_{1 \mathrm{c}}$ assay. However, our findings suggest that identification of people with elevated $2 \mathrm{hPG}$ should be considered for CVD risk stratification either by re-introducing the OGTT for diagnosing prediabetes and diabetes or by other methods $[7,8]$.
Our results support previous findings from the Diabetes Prevention Program (DPP), where individuals with $2 \mathrm{hPG}$ defined prediabetes who reverted to normoglycaemia experienced a concomitant reduction in their cardiovascular risk profile [9]. Previous observations from a general Dutch population also suggested that $2 \mathrm{hPG}$ levels are more strongly associated with all-cause and cardiovascular mortality than
Fig. 1 Rate ratios (RRs) of an event (CVD or death) for reverting from prediabetes to normoglycaemia vs not reverting (a) or for decreasing $1 \mathrm{SD}$ in glycaemic measure over 5 years from phase 7 to phase 9 (b). Grey: unadjusted RR; light blue: adjusting for age and sex; dark blue: further adjusting for previous CVD. The RR for $1 \mathrm{SD}$ decrease (b) is further adjusted for baseline glycaemia in all the analyses. The $x$-axis is on a natural logarithmic scale a

Glycaemic measure

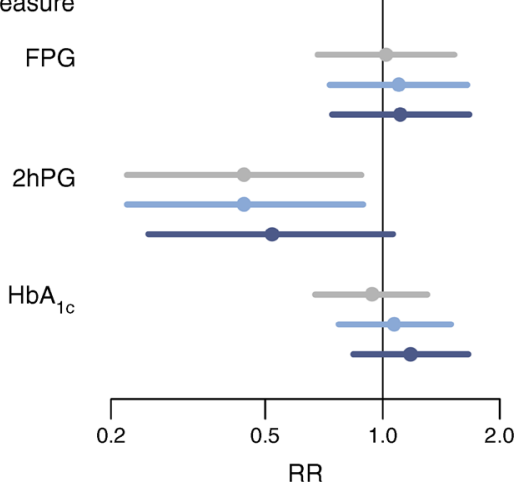

b

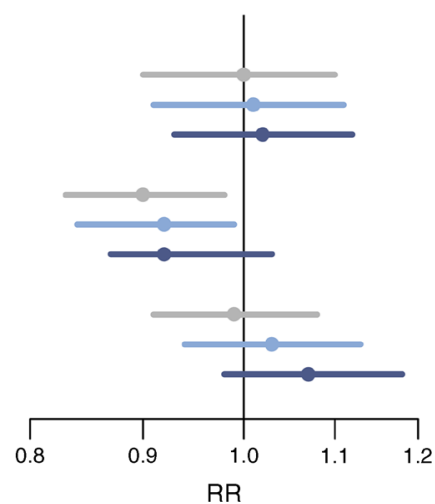


FPG or $\mathrm{HbA}_{1 \mathrm{c}}$ levels in the non-diabetic range [10]. These results may reflect underlying pathophysiological differences in, for example, insulin resistance, which is more pronounced in elevated $2 \mathrm{hPG}$ than in elevated FPG and $\mathrm{HbA}_{1 \mathrm{c}}$ [11].

We found reversion to normoglycaemia from $\mathrm{HbA}_{1 c^{-}}$ defined prediabetes to be less likely than from FPGor $2 \mathrm{hPG}$-defined prediabetes. While our results on reversion rates for FPG- and 2hPG-defined prediabetes are consistent with previous findings [12], evidence on the ability to revert from $\mathrm{HbA}_{1 \mathrm{c}}$-defined prediabetes to normoglycaemia is scarce [12]. A Japanese study reported a reversion rate of $32 \%$ in a study population that was, on average, 10 years younger than the participants of the Whitehall II study [12]. There is less day-today variation in $\mathrm{HbA}_{1 \mathrm{c}}$, and levels in the non-diabetic range may largely be explained by non-glycaemic factors, such as age and ethnicity [13], and may therefore not be modifiable to the same degree as FPG and $2 \mathrm{hPG}$ levels.

We have chosen not to combine different definitions of prediabetes and assess the impact of overall reverting to normoglycaemia. Combining definitions is not in accordance with ADA and IEC and will greatly inflate the prevalence of prediabetes [14]. Furthermore, the corresponding state of normoglycaemia needs to be defined as normal values on all three criteria, which may not be relevant in clinical practice. Alternatively, specific states of prediabetes and normoglycaemia will have to be applied, resulting in numerous transition possibilities for which this study is not powered to examine (ESM Fig. 2 and 3).

Results from the current analysis are strengthened by the longitudinal, well-described, large population size and the validated ascertainment of CVD events. Nevertheless, reversion cannot be ascribed to intervention effects, since the cohort is strictly observational, and all conclusions remain associative and not necessarily causative. There is still controversy between different diabetes organisations with respect to whether $\mathrm{HbA}_{1 \mathrm{c}}$ or FPG should be used to define prediabetes. Our results did not show a reduced risk of CVD or death when people with either $\mathrm{HbA}_{1 \mathrm{c}^{-}}$or FPG-defined prediabetes were able to revert to normoglycemia, based on the respective criterion. On the other hand, we found reversion from $2 \mathrm{hPG}$-defined prediabetes to be associated with a halving of the risk of CVD and death. Although the different diagnostic criteria for prediabetes are likely to remain, the current results would contend that only reversion from 2hPG-defined prediabetes to normoglycaemia is sensitive enough to detect cardiovascular benefit.

Guidelines for people with diabetes are increasingly comprehensive [5], and accordingly, the incidence of complications has dramatically decreased over the past
20 years [15]. In contrast, the lack of accepted guidelines for people with prediabetes has made the prevalence of diabetes-related complications now virtually identical for people with prediabetes vs those with diabetes [16]. The current findings highlight the reduced risk for CVD and death associated with reversion from $2 \mathrm{hPG}$-defined prediabetes, specifically, to normoglycaemia. These findings have important implications for additional cardiovascular risk stratification and intervention in a landscape that has become increasingly controversial [17].

Acknowledgements We thank all participating women and men in the Whitehall II study, as well as all Whitehall II research scientists, study and data managers and clinical and administrative staff who make the study possible. Some of the data were presented as an abstract at the 15 th International Congress of Behavioral Medicine in 2018.

Data availability Whitehall II data, protocols and other metadata are available to the scientific community. Please refer to the Whitehall II data sharing policy at https://www.ucl.ac.uk/whitehallII/data-sharing.

Funding The UK Medical Research Council (K013351, R024227), British Heart Foundation (RG/13/2/30098), and the US National Institutes of Health (R01HL36310, R01AG013196) have supported collection of data in the Whitehall II study.

AH and DRW are supported by the Danish Diabetes Academy, which is funded by an unrestricted grant from the Novo Nordisk Foundation. Support was further provided by the Steno Diabetes Center Aarhus (SDCA) which is partially funded by an unrestricted donation from the Novo Nordisk Foundation. KF is supported by a grant from the Novo Nordisk Foundation. MK is supported by the Medical Research Council (K013351, R024227, S011676), NordForsk, the Academy of Finland (311492) and Helsinki Institute of Life Sciences. The funders of the study had no role in study design, data collection, analysis, interpretation or writing of the report.

Duality of interest KF is funded by the Novo Nordisk Foundation. LP reports personal fees from Novo Nordisk, Merck Astra Zeneca, Boeringer-Ingelheim, Orexigen, Sanofi and Janssen. MK reports research grants from the UK Medical Research Council (K013351, R024227, S011676), NordForsk, Academy of Finland and Helsinki Institute of Life Science during the conduct of the study. MEJ has received research grants from AstraZeneca, AMGEN AB, Sanofi Aventis and BoehringerIngelheim (Investigator-initiated research). The other authors declare no competing interests.

Contribution statement DV and KF contributed to the study concept and design, planned the statistical analyses and drafted the manuscript. DV conducted the statistical analysis. All authors contributed to the interpretation of data. EB, MK, AT and DW contributed to the acquisition of data. All authors revised the manuscript critically and approved the final version of the manuscript. DV and KF are guarantors of the contents of the article and, as such, had full access to all the data in the study and take responsibility for the integrity of the data and the accuracy of the data analysis.

Open Access This article is distributed under the terms of the Creative Commons Attribution 4.0 International License (http:// creativecommons.org/licenses/by/4.0/), which permits unrestricted use, distribution, and reproduction in any medium, provided you give appropriate credit to the original author(s) and the source, provide a link to the Creative Commons license, and indicate if changes were made. 


\section{References}

1. Huang Y, Cai X, Mai W, Li M, Hu Y (2016) Association between prediabetes and risk of cardiovascular disease and all cause mortality: systematic review and meta-analysis. BMJ 355:i5953

2. Vistisen D, Witte DR, Brunner EJ, Kivimaki M, Tabak A, Jorgensen ME et al (2018) Risk of cardiovascular disease and death in individuals with prediabetes defined by different criteria: the Whitehall II study. Diabetes Care 41(4):899-906. https://doi.org/10.2337/dc172530

3. Perreault L, Pan Q, Mather KJ, Watson KE, Hamman RF, Kahn SE (2012) Effect of regression from prediabetes to normal glucose regulation on long-term reduction in diabetes risk: results from the Diabetes Prevention Program Outcomes Study. Lancet. 379(9833):2243-2251. https://doi.org/10.1016/S0140-6736(12) 60525-X

4. Tabák AG, Jokela M, Akbaraly TN, Brunner EJ, Kivimäki M, Witte DR (2009) Trajectories of glycaemia, insulin sensitivity, and insulin secretion before diagnosis of type 2 diabetes: an analysis from the Whitehall II study. Lancet 373(9682):2215-2221. https://doi.org/10.1016/S0140-6736(09)60619-X

5. American Diabetes Association (2018) Classification and diagnosis of diabetes. Sec. 2. In standards of medical care in diabetes - 2018. Diabetes Care 41(Suppl 1):S13-S27. https://doi.org/10.2337/dc18S002

6. Nathan DM, Balkau B, Bonora E, Borch-Johnsen K, Buse JB, Colagiuri $S$ et al (2009) International expert committee report on the role of the A1C assay in the diagnosis of diabetes. Diabetes Care 32(7):1327-1334

7. Cobb J, Eckhart A, Motsinger-Reif A, Carr B, Groop L, Ferrannini $\mathrm{E}(2016) \alpha$-Hydroxybutyric acid is a selective metabolite biomarker of impaired glucose tolerance. Diabetes Care 39(6):988-995. https://doi.org/10.2337/dc15-2752

8. Babbar R, Heni M, Peter A et al (2018) Prediction of glucose tolerance without an oral glucose tolerance test. Front Endocrinol 9(82). https://doi.org/10.3389/fendo.2018.00082

9. Perreault L, Temprosa M, Mather KJ, Horton E, Kitabchi A, Larkin $M$ et al (2014) Regression from prediabetes to normal glucose regulation is associated with reduction in cardiovascular risk: results from the Diabetes Prevention Program Outcomes Study. Diabetes Care 37(9):2622-2631. https://doi.org/10.2337/dc14-0656

10. de Vegt F, Dekker JM, Ruhe HG, Stehouwer CD, Nijpels G, Bouter LM et al (1999) Hyperglycaemia is associated with all-cause and cardiovascular mortality in the Hoorn population: the Hoorn study. Diabetologia. 42(8):926-931. https://doi.org/10.1007/ s001250051249

11. Faerch K, Hulman A, Solomon TP (2016) Heterogeneity of prediabetes and type 2 diabetes: implications for prediction, prevention and treatment responsiveness. Curr Diabetes Rev 12(1):30-41

12. Richter B, Hemmingsen B, Metzendorf MI, Takwoingi Y (2018) Development of type 2 diabetes mellitus in people with intermediate hyperglycaemia. Cochrane Database Syst Rev 10:Cd012661

13. Faerch K, Alssema M, Mela DJ, Borg R, Vistisen D (2018) Relative contributions of preprandial and postprandial glucose exposures, glycemic variability, and non-glycemic factors to $\mathrm{HbA} 1 \mathrm{c}$ in individuals with and without diabetes. Nutr Diabetes 8(1):38. https:// doi.org/10.1038/s41387-018-0047-8

14. Faerch K, Vistisen D (2014) Prevalence of diabetes in Chinese adults. JAMA. 311(2):200. https://doi.org/10.1001/jama.2013. 283127

15. Gregg EW, Li Y, Wang J, Burrows NR, Ali MK, Rolka D et al (2014) Changes in diabetes-related complications in the United States, 1990-2010. N Engl J Med 370(16):1514-1523. https://doi. org/10.1056/NEJMoa1310799

16. Ali MK, Bullard KM, Saydah S, Imperatore G, Gregg EW (2018) Cardiovascular and renal burdens of prediabetes in the USA: analysis of data from serial cross-sectional surveys, 1988-2014. Lancet Diabetes Endocrinol 6(5):392-403. https://doi.org/10.1016/S22138587(18)30027-5

17. Goff DC Jr, Lloyd-Jones DM, Bennett G, Coady S, D'Agostino RB, Gibbons R et al (2014) 2013 ACC/AHA guideline on the assessment of cardiovascular risk: a report of the American College of Cardiology/American Heart Association Task Force on Practice Guidelines. Circulation. 129(25 Suppl 2):S49-S73. https:// doi.org/10.1161/01.cir.0000437741.48606.98

Publisher's note Springer Nature remains neutral with regard to jurisdictional claims in published maps and institutional affiliations. 